\title{
Contactless charge carrier mobility measurement in organic field-effect transistors
}

\author{
W. S. Christian Roelofs, Weiwei Li, Rene A. J. Janssen, Dago M. de Leeuw and Martijn \\ Kemerink
}

\section{Linköping University Post Print}

\section{Tweet}

N.B.: When citing this work, cite the original article.

Original Publication:

W. S. Christian Roelofs, Weiwei Li, Rene A. J. Janssen, Dago M. de Leeuw and Martijn Kemerink, Contactless charge carrier mobility measurement in organic field-effect transistors, 2014, Organic electronics, (15), 11, 2855-2861.

http://dx.doi.org/10.1016/j.orgel.2014.08.027

Copyright: Elsevier http://www.elsevier.com/

Postprint available at: Linköping University Electronic Press http://urn.kb.se/resolve?urn=urn:nbn:se:liu:diva-112628 


\section{Contactless charge carrier mobility measurement in organic field-effect transistors}

W. S. Christian Roelofs, ${ }^{1,2}$ Weiwei Li, ${ }^{1}$ René A. J. Janssen, ${ }^{1}$ Dago M. de Leeuw, ${ }^{3,4}$ and Martijn Kemerink, ${ }^{1,5, a)}$

${ }^{1}$ Eindhoven University of Technology, P.O. Box 513, 5600 MB Eindhoven, The Netherlands

${ }^{2}$ Philips Research Laboratories, High Tech Campus 4, 5656 AE Eindhoven, The Netherlands

${ }^{3}$ Max Planck Institute for Polymer Research, Ackermannweg 10, D- 55128 Mainz, Germany

${ }^{4}$ King Abdulaziz University, Jeddah, Saudi Arabia

${ }^{5}$ Linköping University, Department of Physics, Chemistry and Biology (IFM), SE-581 83 Linköping, Sweden

a) Electronic mail: martijn.kemerink@liu.se

Key words: mobility, contact resistance, organic field-effect transistor, impedance spectroscopy, polymer semiconductor.

Abstract

With the increasing performance of organic semiconductors, contact resistances become an almost fundamental problem, obstructing the accurate measurement of charge carrier mobilities. Here, a generally applicable method is presented to determine the true charge carrier mobility in an organic field-effect transistor (OFET). The method uses two additional finger-shaped gates that capacitively generate and probe an alternating current in the OFET channel. The time lag between drive and probe can directly be related to the mobility, as is shown experimentally and numerically. As the scheme does not require the injection or uptake of charges it is fundamentally insensitive to contact resistances. Particularly for ambipolar materials the true mobilities are found to be substantially larger than determined by conventional (direct current) schemes. 


\section{Introduction}

The performance of organic field-effect transistors (OFET) increases remarkably, and an increasing number of organic semiconductors is being reported with a charge carrier mobility over that of a-Si, $\sim 1 \mathrm{~cm}^{2} / \mathrm{Vs}$. [1-2] Especially n-type and ambipolar polymers recently made strong progression.[3-8] However, as the channel resistance decreases due to the increase of organic semiconductor mobilities, contact resistances often become the bottleneck for the total device performance.[1, 9-10] Many strategies have been reported to decrease the contact resistance for either holes or electrons,[1, 10-11] using self-assembled monolayers,[12-13] doping,[14-15] interlayers,[16-17] or by changing the device lay-out.[9, 18] Contact resistance is an (even) more fundamental problem in ambipolar transistors, where both electrons and holes need to be injected. [5, 19] The electron and hole injection barriers at any contact always sum up to the semiconductor band gap. Hence the injection barrier cannot be negligible for both holes and electrons simultaneously, leading to a contact resistance for at least one of the two charges. By using different contact materials for source and drain this problem can partially be solved, but for ease of fabrication it is preferred to have a single electrode material that is able to inject both types of charge carriers.[20]

For research purposes one is often only interested in the transport properties of the semiconductor. The charge carrier mobility is then typically obtained by measuring the current in an OFET.[21] When the transistor suffers from contact resistance, a lower current and concomitantly a lower mobility is found. Correction for contact resistance is possible by estimating its value.[22-26] The most popular way to do so is by the transfer line method in which the resistance of OFETs as function of the channel length is measured. The extrapolated resistance at zero channel length is a measure for (twice) the contact resistance. This method however requires good device reproducibility.[27-28]

In view of the above there is a clear need for a tool to determine the charge carrier mobility that is insensitive to contact resistance. Here we present such a technique. The basic idea is that an alternating current (AC) is capacitively generated and probed in an OFET channel. To this end two additional finger gates are placed near the 
accumulation layer. Conventional source and drain contacts are still required to fill the accumulation layer but do not need to absorb or inject any current and are therefore decoupled from the mobility measurement.

\section{Materials and methods}

\section{Device fabrication}

Devices were fabricated on cleaned glass substrates. The finger gates were created by evaporating $40 \mathrm{~nm}$ thick Au electrodes through a shadow mask substrate, preceded by a $2 \mathrm{~nm} \mathrm{Cr}$ adhesion layer. Subsequently a $870 \mathrm{~nm}$ thick Cytop dielectric was applied by spin coating, followed by annealing for $30 \mathrm{~min}$. at $150{ }^{\circ} \mathrm{C}$. On top of that, 40 $\mathrm{nm} \mathrm{Au}$ source and drain electrodes were evaporated through a shadow mask. The active layer, poly[2,5-bis(2hexyldecyl)-2,3,5,6-tetrahydro-3,6-dioxopyrrolo[3,4-c]pyrrole-1,4-diyl-alt-[2,2'-(1,4-phenylene)bisthiophene]-5,5'diyl] (PDPPTPT), was applied by spin coating from a chloroform solution and annealed for $30 \mathrm{~min}$. at 200 ${ }^{\circ} \mathrm{C}$. [29-30] Prior to spin coating, the Cytop dielectric required a treatment with a mild nitrogen plasma to enhance the semiconductor wetting. The gold work function was modified by either applying the semiconductor immediately after the plasma treatment, or first rinsing the gold electrodes with isopropanol. A second Cytop layer was spin coated on top of the stack and annealed at $150{ }^{\circ} \mathrm{C}$. for $30 \mathrm{~min}$. forming the gate dielectric. The thickness of the dielectric was $1.10 \mu \mathrm{m}$ and $0.85 \mu \mathrm{m}$ for the device with the solvent-rinsed contacts and non-rinsed contacts, resulting in a gate capacitance of $C_{G}=1.85 \mathrm{nC} / \mathrm{cm}^{2}$ and $C_{G}=2.4 \mathrm{nC} / \mathrm{cm}^{2}$, respectively. To finish the device, a $40 \mathrm{~nm}$ $\mathrm{Au}$ gate electrode was evaporated through a shadow mask. OFETs were measured in a high vacuum $\left(10^{-5} \mathrm{mbar}\right)$ probe station. Transfer characteristics were measured with a Keithley 4200 source-measure unit. Impedance spectroscopy was performed using a Solartron 1260A impedance analyzer in combination with a Keithley 2636 to apply the DC gate bias. The resulting lay-out of our device is shown in Fig. 1a.

\section{AC characterization method}

The device consists of a regular bottom-contact top-gate OFET, with the addition of a pair of finger gates positioned below the transistor channel. A dielectric separates the fingers from the transistor channel to ensure that their influence on the charges in the channel is entirely capacitive. The channel formed between the short 
distance between a pair of source and drain electrodes is used for simultaneous conventional direct-current (DC)OFET characterization, whereas the long distance channel is used for AC characterization.

a)

\begin{tabular}{|c|c|c|}
\hline \multicolumn{3}{|c|}{ Au, Gate } \\
\hline \multicolumn{3}{|c|}{ Cytop, Dielectric } \\
\hline$\mathrm { Au } \longdiv { \mathrm { Au } }$ & DPP polymer, Semiconductor & $\mathrm{Au} \cap \mathrm{Au}$ \\
\hline Source Drair & Cytop, Dielectric & Source Drain \\
\hline & Glass, Substrate Finger gates & \\
\hline
\end{tabular}

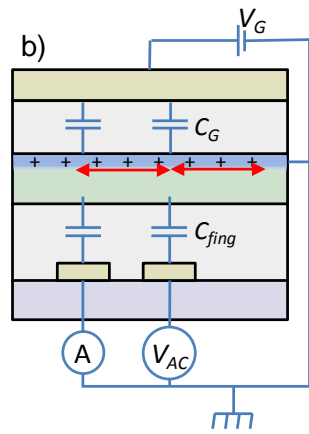

Figure 1: a) Cross section of the device lay-out. b) Schematic operation principle of the finger gate structure in hole accumulation (i.e. at negative gate bias $V_{G}$ ). The accumulation layer is grounded via the source and drain.

As shown in Fig. 1b an AC-bias is applied to one of the finger electrodes. The bias alternatingly pushes charges away from, or pulls charges towards the channel region above the finger gate, generating charge waves in the transistor channel. These charge waves are probed capacitively by the second finger gate. The time lag between the drive and probe signals can be related to the charge carrier mobility in the channel above the finger gates. In particular, performing impedance spectroscopy on the fingers, a characteristic frequency $f_{c}$ can be observed. Its value corresponds to the reciprocal transit time of charges moving between the fingers. We define the characteristic frequency of the finger-gate system as the frequency where the conductance divided by the frequency $f$ peaks. The physical meaning of this quantity is the charge current per oscillation period flowing between the finger gate electrodes.

It is important that the modulating finger gates are sufficiently far away from the source and drain contacts such that the charge density modulations have damped out at the contacts. Then the contacts do not absorb or inject any charges and do not play a role in the AC measurement. With this condition fulfilled, this method differs fundamentally from conventional impedance measurements in which the source and drain do need to absorb and inject charge.[31-35] 


\section{Theory}

In order to determine the relation between characteristic frequency and mobility, the system will first be analyzed analytically for which it is simplified to the four capacitor circuit as drawn in Fig. 1b. Furthermore, the charge flow away from the finger gate into the rest of the transistor channel is neglected. On basis of a simple dimension analysis the RC-time $\tau_{R C}$ of the system may be expected to scale as:

$$
\tau_{R C} \propto R W L_{\text {fing }}\left(C_{\text {fing }}+C_{G}\right) \text {, }
$$

with $R$ the resistance of the accumulation channel, $C_{G}$ the gate capacitance per unit area, $C_{f i n g}$ the finger gate capacitance per unit area, $W$ the finger width, and $L_{\text {fing }}$ the length of a finger gate electrode. The resistance $R$ is given by:

$$
R=\frac{L}{\sigma W}
$$

where $\sigma$ is the conductivity of the $2 \mathrm{D}$ accumulation layer with the unit $S$. The distance $L$ is the length that charges travel from one finger gate to the other finger gate, which is expected to be proportional to the finger gate spacing $L_{s p}$ plus a fraction $x$ of the finger width $L_{\text {fing }}: L=L_{s p}+x L_{\text {fing. }}$. The fraction $x$ depends on the device geometry and shall be found numerically below. The conductivity of the accumulation channel $\sigma$ is proportional to the amount of accumulated charges and their mobility $\mu$ :

$$
\sigma=\left(V_{G}-V_{T}\right) C_{G} \mu
$$

with $V_{G}$ the gate bias and $V_{T}$ the threshold voltage.

The characteristic frequency is expected to scale with the estimated RC-time as $f_{c} \propto 1 / \tau_{R C}$. Combining this with Eqs. 1-3 yields:

$$
f_{c} \propto \frac{\left(V_{G}-V_{T}\right) \mu}{L_{\text {fing }} L} \frac{C_{G}}{\left(C_{G}+C_{\text {fing }}\right)} .
$$

To find the exact value of the characteristic frequency we analyzed the entire system numerically. Impedance spectroscopy on the finger gates is simulated on a 1D grid by forward time integration. The finger-gate system is considered to be a resistor-capacitor (RC)-network. The transistor channel is split in $N$ separate RC-elements as shown in Fig. 2a. The fingers are placed at distinct grid cells $i$ with a finger capacitance $C_{\text {fing,i. }}$ At positions where no 
finger is located the finger capacitance is set to zero. A time-varying finger gate bias $V_{\text {fing, }}(t)$ is set to one of the fingers, the other finger is grounded and the time dependent current flowing to this finger gate is tracked. A constant mobility $\mu$ is assumed, although experimentally the mobility is charge density dependent. This assumption does not change the outcome as long as the variation in charge density is small compared to the average charge density in the channel. Initially the potential is assumed to be constant in every grid cell, which is set by the source and the drain bias, $V_{D}=V_{S}=0 \mathrm{~V}$. The potential and charge density in each grid cell $p_{i}$ are related via the capacitance as:

$$
p_{i}=C_{G}\left(V_{G}+V_{i}\right)+C_{\text {fing }, i}\left(V_{\text {fing }, i}+V_{i}\right)
$$

where $V_{G}$ is the gate bias, $C_{G}$ the gate capacitance and $V_{i}$ the potential in the channel at grid cell $i$. Using the continuity equation the (change in) charge carrier density in each grid cell is calculated after each time step $\Delta t$ :

$$
q \frac{\Delta p_{i}}{\Delta t}=\frac{\Delta j_{i}}{\Delta x}
$$

with $q$ the elementary charge, $\Delta x$ the distance between subsequent grid cells, and $j$ the current. The current follows from Ohm's law:

$$
j_{i, i+1}=q p_{i} \mu \frac{\left(V_{i+1}-V_{i}\right)}{\Delta x}
$$

Finally, after each time step new channel potentials are calculated using Eq. (4). The densities at the source and drain contacts are kept constant in time, which represents Ohmic injection. We note however that the results do not change by increasing the distance between the contacts and the finger structure, indicating that the choice of the contacts does not influence the outcome of the simulations. The AC experiment is performed after giving the accumulation layer enough time to be filled by the contacts and the system has found its equilibrium.

From the numerical simulations the following relation was found:

$$
f_{c}=\frac{\left(V_{G}-V_{T}\right) \mu}{2 L_{f i n g} L} \frac{C_{G}}{\left(C_{G}+C_{f i n g}\right)}
$$



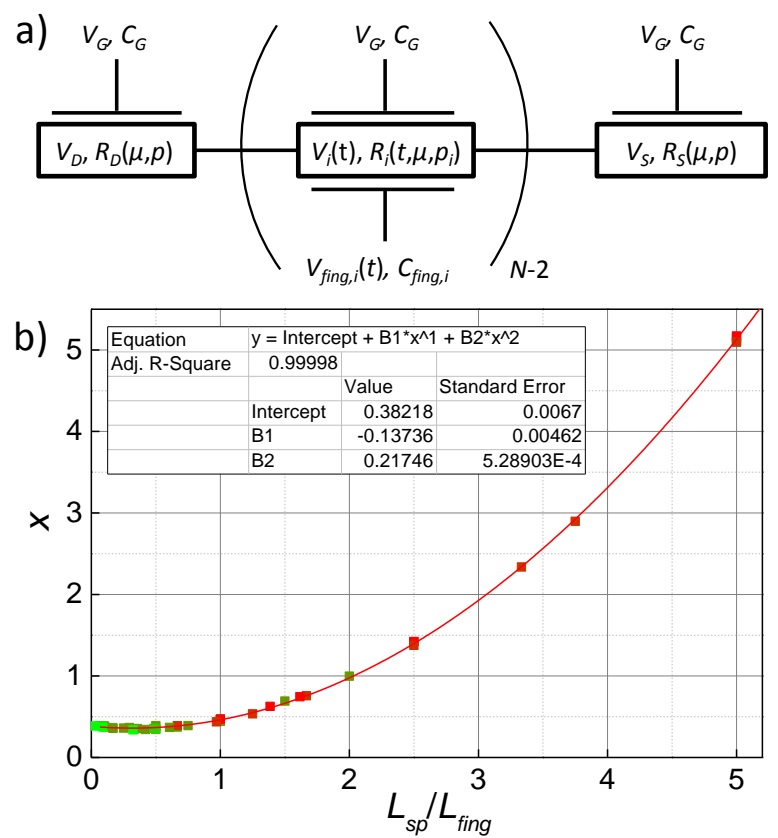

Figure 2: a) Schematic drawing of the simulation of the finger gate system. b) The fraction $x$ in $L=L_{s p}+x L_{\text {fing }}$ as function of the ratio of the width of the inter-finger gap finger, $L_{s p}$, over the width of the fingers, $L_{f i n g}$. For small values of $L_{s p} / L_{f i n g} x=0.38$. Within numerical accuracy the points can be parameterized by a second order polynomial as shown by the red line; the legend provides the coefficients. The different colors refer to different lengths of the finger gates $L_{s p}$ ranging from $20 \mu \mathrm{m}$ (green) to 300 $\mu \mathrm{m}$ (red).

The dependence of $x$ on the device geometry $\left(L_{s p} / L_{f i n g}\right)$ is shown in Fig. 2b. For a high signal to noise ratio, the finger length is preferably larger than the finger spacing. In this region, when $L_{s p}<L_{\text {fing, }}$ the fraction $x$ was numerically found to be equal to 0.38 . With all parameters in Eq. 4 known, the characteristic frequency of the system allows one to determine the charge mobility. The parameterization of $x$ makes the method generally applicable for any geometry that fulfills the criterion that the finger gates are far away from the source and drain contacts.

\section{Experimental results}

\section{Transistor characteristics}

Before turning to the AC mobility measurement, we shall first illustrate the urgency to avoid injection barriers by conventionally measuring the mobility in transistors with source and drain contacts having different work 
functions, but based on the same DPP-polymer (PDPPTPT). Different work functions were established by using plasma cleaned gold and solvent cleaned gold. Rinsing gold contacts by a solvent is known to decrease their work function from the range of $-(5.0-5.5) \mathrm{eV}$ for plasma cleaned gold to the range of $-(4.7-4.9)$ eV for solvent cleaned gold.[36] The HOMO and LUMO levels of PDPPTPT were earlier determined with cyclic voltammetry to be -5.35 and $-3.53 \mathrm{eV}$, respectively.[29] Based on these numbers, a hole injection barrier may be expected when using solvent cleaned gold and not when using plasma cleaned gold. In Fig. 3a the output curves of the two transistors are shown for negative gate biases, i.e. hole accumulation. The drain current of the transistor with the low workfunction contacts $\left(\varphi_{2}\right)$ shows a clear s-shape indicative for a hole injection barrier.[37] This is not observed in the transistor with the higher work-function contacts $\left(\varphi_{1}\right)$. Transfer curves of the OFETs are presented in Fig. $3 \mathrm{~b}$, where the gate bias is multiplied by the actual gate capacitance for fair comparison between transistors with different dielectric thicknesses. The transistor with the high work-function contacts $\left(\varphi_{1}\right)$ shows unipolar hole transport. On basis of the inset to Fig. 3a the negligible electron injection can be rationalized. The linear hole mobility extracted from the transfer curve amounts to $0.28 \pm 0.02 \mathrm{~cm}^{2} / \mathrm{Vs}$. The mobility is higher than obtained earlier for PDPPTPT, $[19,29,38]$ because of a different gate dielectric and an improved synthesis of the polymer.[30] The transistor with the low work-function contacts $\left(\varphi_{2}\right)$ shows ambipolar charge transport, but the hole currents at negative gate biases are smaller than those observed in the transistor with the high work-function contacts. Extracted hole and electron mobilities in this case amount to $0.25 \pm 0.02$ and $0.04 \pm 0.02 \mathrm{~cm}^{2} / \mathrm{Vs}$. The uncertainty in the electron mobility is relatively large due to gate leakage currents. As explained above, the price paid for lowering the electron injection barrier and enabling ambipolar operation is the formation of a hole injection barrier. At negative gate bias this lowers the transistor current and consequently a lower (apparent) hole mobility is found for this device. Furthermore, a higher gate bias is needed to reach this mobility, leading to an apparent shift in threshold voltage. 

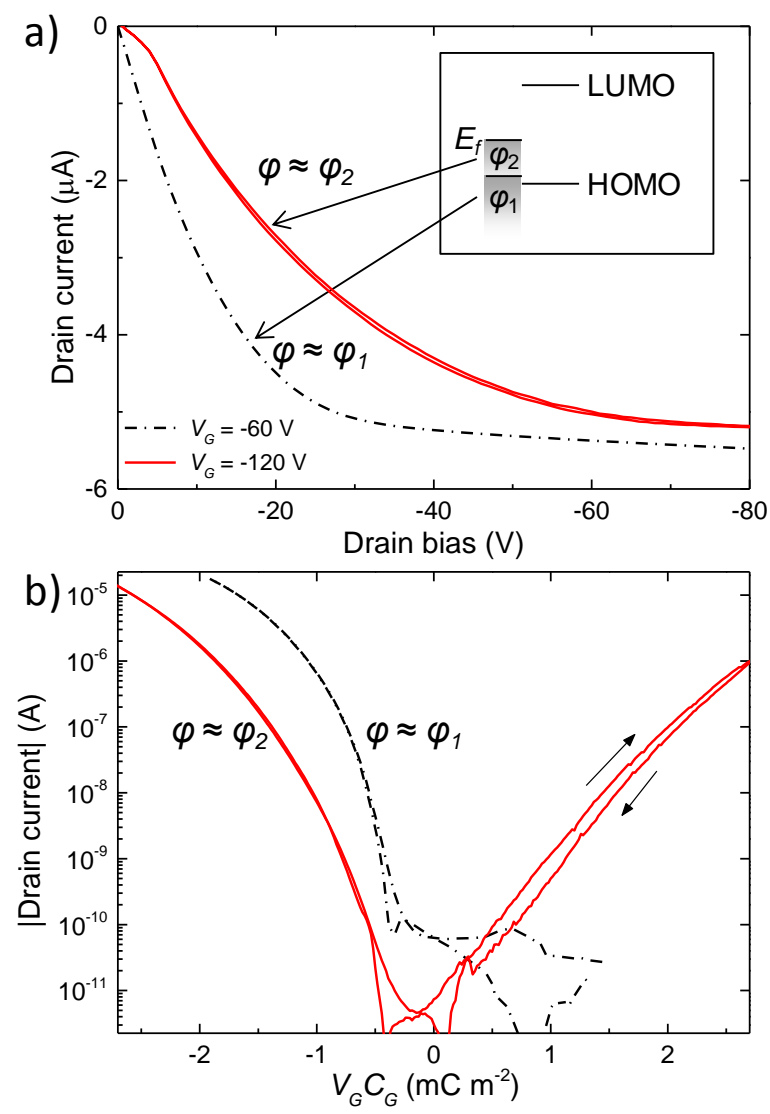

Figure 3: a) Output curve of an OFET with gold contacts and an OFET with gold contacts rinsed with isopropanol. Solvent rinsing slightly decreases the gold work function, resulting in a less deep work function $\left(\varphi_{2}\right)$ than for plasma cleaned gold $\left(\varphi_{1}\right)$. $L_{F E T}=30 \mu \mathrm{m}, W_{F E T}=1 \mathrm{~mm}$. Inset: Schematic energy diagram of the metal-semiconductor interface for three different workfunctions $\varphi$, illustrating the position of the Fermi level with respect to the HOMO and LUMO level. b) Transfer characteristics of the two transistors at a drain bias of $-40 \mathrm{~V}$.

\section{Contactless mobility measurement}

We shall now turn to the contactless mobility measurement. Impedance spectroscopy was performed on a finger-gate structure on the same substrate on which also the ambipolar OFETs of Fig. 3 were measured. The source and drain contacts far away from the modulated region were grounded to fix the chemical potential in the channel. Gate biases varying from -150 to $-65 \mathrm{~V}$ and from 200 to $150 \mathrm{~V}$ were applied to create hole or electron accumulation channels, respectively. Note that accumulation layers can be filled completely despite the presence of charge injection barriers. In steady state no current is flowing in the transistor channel and therefore no charges are injected or extracted at the contacts. The finger-gate system was biased by a $3 \mathrm{~V} A C$-bias applied to a finger 
and sweeping its frequency from $1 \mathrm{kHz}$ to $500 \mathrm{kHz}$, while monitoring the current at the grounded second finger gate. The resulting conductance-over- $f$ data are shown in Fig. 4a. An impedance signal could be measured both for negative gate biases where holes are accumulated as well as for positive gate biases where electrons are accumulated. The conductance-over- $f$ shows a clear maximum at the characteristic frequency of the finger-gate system. A shift of the conductance spectrum to higher frequencies is observed as the absolute gate bias increases, in accordance with Eq. 8. The characteristic frequency, here taken as the position of the maximum, as function of gate bias is plotted in Fig. 4b. In Figs. $4 c$ and $4 d$ the impedance results are compared with the numerical simulations. To show that a meaningful characteristic frequency can be defined also from experiments all data in Figs. $4 \mathrm{a}$ and $4 \mathrm{~b}$ are collapsed on single curves in Fig. $4 \mathrm{c}$ and $4 \mathrm{~d}$ by normalizing the frequency by the characteristic frequency. The drawn lines represent the simulation results and the dots represent the measured data. An excellent qualitative and quantitative agreement is found with the numerical simulation for all measured gate biases. 

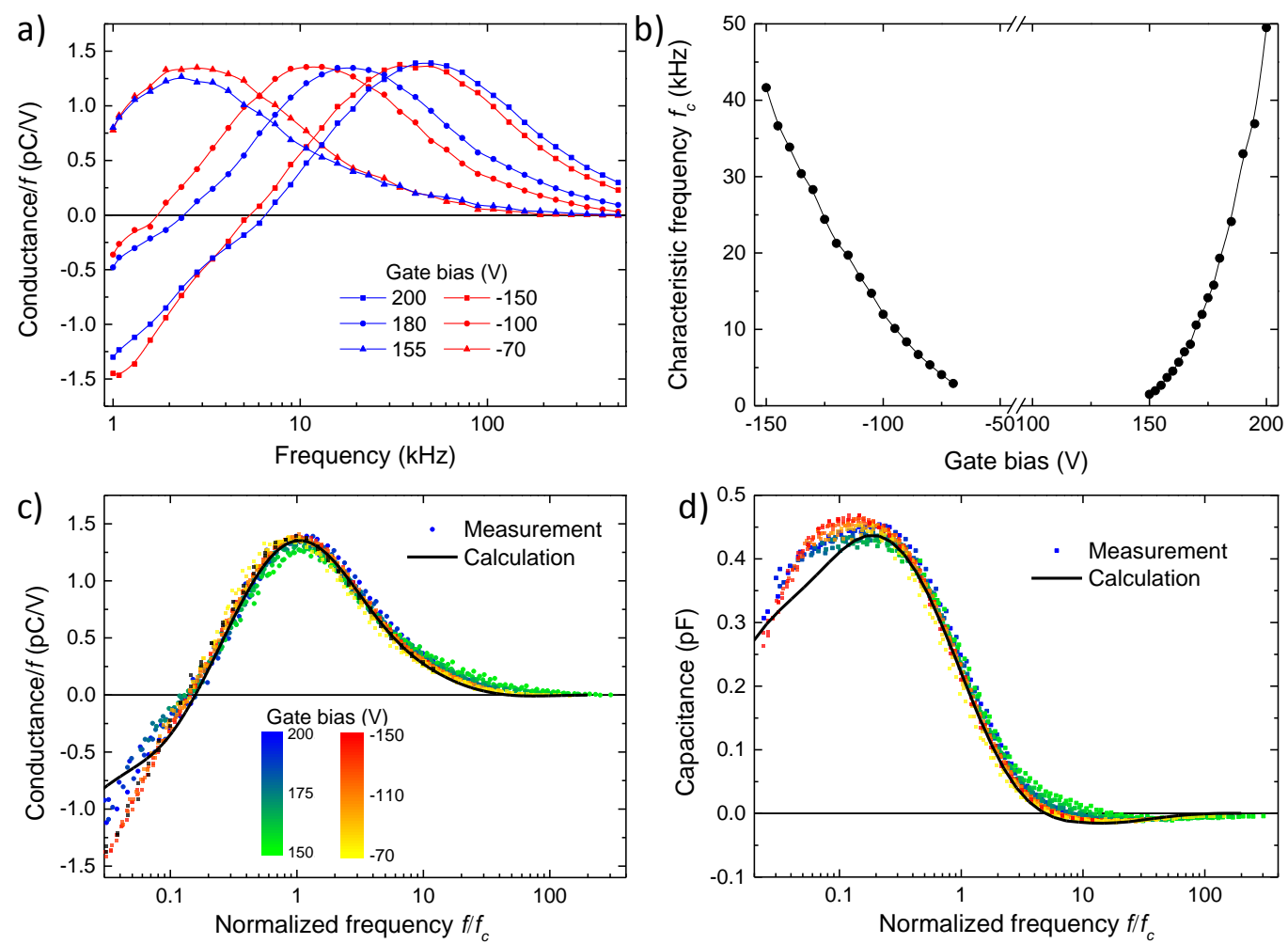

Figure 4: Impedance spectroscopy results. a) Conductance-over-f for various gate biases. b) Characteristic frequency, defined as the maximum of conductance over $f$, as function of gate bias. c,d) Measured and calculated conductance-over- $f$ c) and capacitance d) as function of the normalized frequency. The color bars indicate the applied gate bias. $L_{\text {fing }}=160 \mu \mathrm{m}$ and $L_{s p}$ $=50 \mu \mathrm{m}$.

The mobility can now straightforwardly be extracted from the position of the characteristic frequency. The charge carrier mobility in transistors is usually determined from the transfer characteristics by measuring the change in drain current $d l_{d}$ upon a small change in the gate bias $d V_{G}$ using:

$$
\mu_{l i n}=\frac{d l_{d}}{d V_{G}} \frac{L_{F E T}}{W_{F E T} C_{G} V_{d}}
$$

with $V_{d}$ the drain bias, $L_{F E T}$ the length and $W_{F E T}$ the width of the transistor channel. This method avoids errors due to uncertainties in the threshold voltage. For a fair comparison, the mobility from the impedance data has to be extracted similarly. The mobility will therefore be extracted by calculating the change in characteristic frequency $d f_{c}$ upon a small change in the gate bias by differentiating Eq. 8: 


$$
\mu_{\text {imp }}=\frac{d f_{c}}{d V_{G}} \frac{2 L_{\text {fing }} L\left(C_{G}+C_{\text {fing }}\right)}{C_{G}} .
$$

The mobilities extracted from the measured impedance spectra are plotted in Fig. 5 together with the linear mobilities extracted from the transfer curves of Fig. 3a. Maximum mobilities extracted by impedance spectroscopy on the finger gates amount to 0.46 and $1.08 \mathrm{~cm}^{2} / \mathrm{Vs}$ for holes and electrons, respectively. Both methods show an increase in mobility with increasing charge density. For the region where they overlap the hole mobilities extracted by impedance spectroscopy are similar to the values found in conventional DC-characterization of the transistor without hole injection barriers. However, the transistor with a hole injection barrier shows only comparable mobilities at significantly higher gate biases. A gate field is known to assist charge injection and therefore contact resistance plays a smaller role at high gate biases.[37]

Since electrons suffer from a larger injection barrier, the effect for electrons is even larger. A 20 times higher mobility is found with impedance spectroscopy than obtained from the DC transfer curves. We have to note that this value may still be underestimated due to a gate leakage current. The leakage may cause some accumulated charges to leak away, effectively decreasing the accumulated charge density. Nonetheless, it clearly shows that due to the contact resistance the electron mobility was highly underestimated by extracting the mobility from the transfer characteristics. With impedance spectroscopy on the finger-gate system, the mobility measurement is decoupled from charge injection and the true mobility in the transistor channel could be extracted. In some devices where record mobilities are measured, also a contact resistance is present. [4] Likely these mobilities are therefore underestimated and higher values may be obtained with the method presented in this paper. 


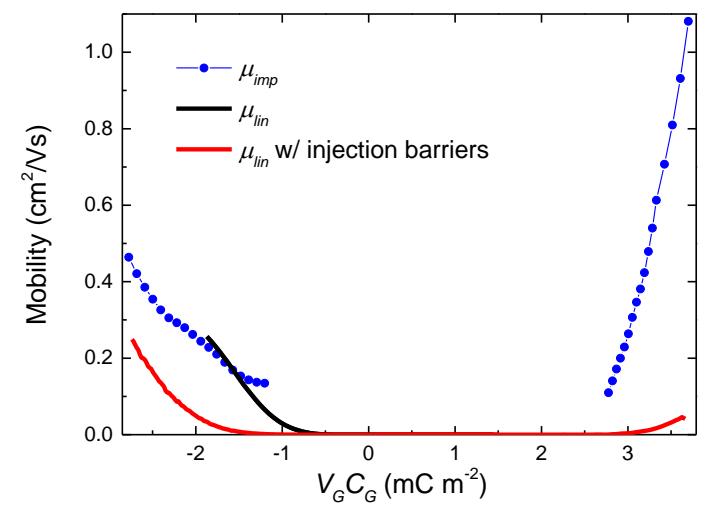

Figure 5: Extracted charge mobilities using impedance spectroscopy (symbols) as function of gate bias. The mobilities extracted from the transfer curves of Fig. $1 \mathrm{~b}$ are presented as solid lines.

\section{Conclusion}

We have presented a general applicable method to measure charge carrier mobilities in organic field-effect transistors, independent of contact resistance. By using capacitive generation and detection of alternating currents there is no need to inject and/or extract charges during the mobility measurement and contact resistance effects are avoided. For that, two additional finger gates were positioned near the accumulation channel. By performing impedance spectroscopy on the finger gates the true mobility could be determined. Comparison to mobilities measured in a conventional (DC) manner confirmed that due to contact resistance the latter present a lower limit. For our devices, the underestimation of the actual mobility went up to a factor 20 for electrons in an ambipolar device. Expressions are provided to allow application of the method to systems with arbitrary dimensions. 


\section{References}

[1] H. Dong, X. Fu, J. Liu, Z. Wang, W. Hu, 25th Anniversary Article: Key Points for High-Mobility Organic FieldEffect Transistors, Adv. Mater., 25 (2013) 6158-6183.

[2] A. Facchetti, Organic semiconductors: Made to order, Nat. Mater., 12 (2013) 598-600.

[3] C.B. Nielsen, M. Turbiez, I. McCulloch, Recent Advances in the Development of Semiconducting DPPContaining Polymers for Transistor Applications, Adv. Mater., 25 (2013) 1859-1880.

[4] B. Sun, W. Hong, Z. Yan, H. Aziz, Y. Li, Record High Electron Mobility of $6.3 \mathrm{~cm}^{2} \mathrm{~V}^{-1} \mathrm{~s}^{-1}$ Achieved for Polymer Semiconductors Using a New Building Block, Adv. Mater., 26 (2014) 2636-2642.

[5] Y. Zhao, Y. Guo, Y. Liu, 25th Anniversary Article: Recent Advances in n-Type and Ambipolar Organic FieldEffect Transistors, Adv. Mater., 25 (2013) 5372-5391.

[6] J. Lee, A.R. Han, H. Yu, T.J. Shin, C. Yang, J.H. Oh, Boosting the Ambipolar Performance of Solution-Processable Polymer Semiconductors via Hybrid Side-Chain Engineering, J. Am. Chem. Soc., 135 (2013) 9540-9547.

[7] W. Li, K.H. Hendriks, W.S.C. Roelofs, Y. Kim, M.M. Wienk, R.A.J. Janssen, Efficient Small Bandgap Polymer Solar Cells with High Fill Factors for 300 nm Thick Films, Adv. Mater., 25 (2013) 3182-3186.

[8] J. Mei, Y. Diao, A.L. Appleton, L. Fang, Z. Bao, Integrated Materials Design of Organic Semiconductors for FieldEffect Transistors, J. Am. Chem. Soc., 135 (2013) 6724-6746.

[9] M. Gruber, E. Zojer, F. Schürrer, K. Zojer, Impact of Materials versus Geometric Parameters on the Contact Resistance in Organic Thin-Film Transistors, Adv. Funct. Mater., 23 (2013) 2941-2952.

[10] D. Natali, M. Caironi, Charge Injection in Solution-Processed Organic Field-Effect Transistors: Physics, Models and Characterization Methods, Adv. Mater., 24 (2012) 1357-1387.

[11] H. Ma, H.-L. Yip, F. Huang, A.K.Y. Jen, Interface Engineering for Organic Electronics, Adv. Funct. Mater., 20 (2010) 1371-1388.

[12] S.A. DiBenedetto, A. Facchetti, M.A. Ratner, T.J. Marks, Molecular Self-Assembled Monolayers and Multilayers for Organic and Unconventional Inorganic Thin-Film Transistor Applications, Adv. Mater., 21 (2009) 1407-1433.

[13] N. Kawasaki, Y. Ohta, Y. Kubozono, A. Fujiwara, Hole-injection barrier in pentacene field-effect transistor with Au electrodes modified by $\mathrm{C}_{16} \mathrm{H}_{33} \mathrm{SH}$, Appl. Phys. Lett., 91 (2007) 123518.

[14] F. Ante, D. Kälblein, U. Zschieschang, T.W. Canzler, A. Werner, K. Takimiya, M. Ikeda, T. Sekitani, T. Someya, H. Klauk, Contact Doping and Ultrathin Gate Dielectrics for Nanoscale Organic Thin-Film Transistors, Small, 7 (2011) 1186-1191.

[15] C. Vanoni, S. Tsujino, T.A. Jung, Reduction of the contact resistance by doping in pentacene few monolayers thin film transistors and self-assembled nanocrystals, Appl. Phys. Lett., 90 (2007) 193119.

[16] Y. Zhou, C. Fuentes-Hernandez, J. Shim, J. Meyer, A.J. Giordano, H. Li, P. Winget, T. Papadopoulos, H. Cheun, J. Kim, M. Fenoll, A. Dindar, W. Haske, E. Najafabadi, T.M. Khan, H. Sojoudi, S. Barlow, S. Graham, J.-L. Brédas, S.R. Marder, A. Kahn, B. Kippelen, A Universal Method to Produce Low-Work Function Electrodes for Organic Electronics, Science, 336 (2012) 327-332.

[17] M.C. Gwinner, Y. Vaynzof, K.K. Banger, P.K.H. Ho, R.H. Friend, H. Sirringhaus, Solution Processed Zinc Oxide as High Performance Air Stable Electron Injector in Organic Ambipolar Light Emitting Field Effect Transistors, Adv. Funct. Mater., 20 (2010) 3457-6922.

[18] Y. Xu, P. Darmawan, C. Liu, Y. Li, T. Minari, G. Ghibaudo, K. Tsukagoshi, Tunable contact resistance in doublegate organic field-effect transistors, Org. Electron., 13 (2012) 1583-1588.

[19] W.S.C. Roelofs, W.H. Adriaans, R.A.J. Janssen, M. Kemerink, D.M. de Leeuw, Light Emission in the Unipolar Regime of Ambipolar Organic Field-Effect Transistors, Adv. Funct. Mater., 23 (2013) 4133-4139.

[20] K.-J. Baeg, J. Kim, D. Khim, M. Caironi, D.-Y. Kim, I.-K. You, J.R. Quinn, A. Facchetti, Y.-Y. Noh, Charge Injection Engineering of Ambipolar Field-Effect Transistors for High-Performance Organic Complementary Circuits, ACS Appl. Mater. Interfaces, 3 (2011) 3205-3214.

[21] S. Tiwari, N.C. Greenham, Charge mobility measurement techniques in organic semiconductors, Opt. Quant. Electron., 41 (2009) 69-89.

[22] S.D. Wang, T. Minari, T. Miyadera, K. Tsukagoshi, Y. Aoyagi, Contact-metal dependent current injection in pentacene thin-film transistors, Appl. Phys. Lett., 91 (2007) 203508. 
[23] M. Imakawa, K. Sawabe, Y. Yomogida, Y. Iwasa, T. Takenobu, Extraction of the contact resistance from the saturation region of rubrene single-crystal transistors, Appl. Phys. Lett., 99 (2011) 233301.

[24] C. Reese, Z. Bao, Detailed Characterization of Contact Resistance, Gate-Bias-Dependent Field-Effect Mobility, and Short-Channel Effects with Microscale Elastomeric Single-Crystal Field-Effect Transistors, Adv. Funct. Mater., 19 (2009) 763-771.

[25] G. Horowitz, P. Lang, M. Mottaghi, H. Aubin, Extracting Parameters from the Current-Voltage Characteristics of Organic Field-Effect Transistors, Adv. Funct. Mater., 14 (2004) 1069-1074.

[26] T.J. Richards, H. Sirringhaus, Analysis of the contact resistance in staggered, top-gate organic field-effect transistors, J. Appl. Phys., 102 (2007) 094510.

[27] J.H. Klootwijk, C.E. Timmering, Merits and limitations of circular TLM structures for contact resistance determination for novel III-V HBTs, Microelectronic Test Structures, 2004. Proceedings. ICMTS '04. The International Conference on, (2004) 247-252.

[28] D.K. Schroder, Semiconductor material and device characterization, John Wiley \& Sons2006.

[29] J.C. Bijleveld, V.S. Gevaerts, D. Di Nuzzo, M. Turbiez, S.G.J. Mathijssen, D.M. de Leeuw, M.M. Wienk, R.A.J. Janssen, Efficient solar cells based on an easily accessible diketopyrrolopyrrole polymer, Adv. Mater., 22 (2010) E242.

[30] K.H. Hendriks, G.H.L. Heintges, V.S. Gevaerts, M.M. Wienk, R.A.J. Janssen, High-Molecular-Weight Regular Alternating Diketopyrrolopyrrole-based Terpolymers for Efficient Organic Solar Cells, Angew. Chem., Int. Ed., 52 (2013) 8341-8344.

[31] K. Ryu, I. Kymissis, V. Bulovic, C.G. Sodini, Direct extraction of mobility in pentacene OFETs using C-V and I-V measurements, Electron Device Letters, IEEE, 26 (2005) 716-718.

[32] J. Lin, M. Weis, D. Taguchi, T. Manaka, M. Iwamoto, Carrier injection and transport in organic field-effect transistor investigated by impedance spectroscopy, Thin Solid Films, 518 (2009) 448-451.

[33] G.H. Gelinck, E. van Veenendaal, H. van der Vegte, R. Coehoorn, Capacitance-voltage characteristics of organic thin-film transistors, Proc. SPIE 6658, Organic Field-Effect Transistors VI, 6658 (2007) 665802-665809.

[34] H.H.P. Gommans, M. Kemerink, G.G. Andersson, R.M.T. Pijper, Charge transport and trapping in Cs-doped poly(dialkoxy-p-phenylene vinylene) light-emitting diodes, Phys. Rev. B, 69 (2004) 155216.

[35] M.J. Deen, M.H. Kazemeini, S. Holdcroft, Contact effects and extraction of intrinsic parameters in poly(3alkylthiophene) thin film field-effect transistors, J. Appl. Phys., 103 (2008) 124509.

[36] Z. Chen, M.J. Lee, R. Shahid Ashraf, Y. Gu, S. Albert-Seifried, M. Meedom Nielsen, B. Schroeder, T.D. Anthopoulos, M. Heeney, I. McCulloch, H. Sirringhaus, High-Performance Ambipolar DiketopyrrolopyrroleThieno[3,2-b]thiophene Copolymer Field-Effect Transistors with Balanced Hole and Electron Mobilities, Adv. Mater., 24 (2012) 647-652.

[37] J.J. Brondijk, F. Torricelli, E.C.P. Smits, P.W.M. Blom, D.M. de Leeuw, Gate-bias assisted charge injection in organic field-effect transistors, Org. Electron., 13 (2012) 1526-1513.

[38] W.S.C. Roelofs, M.-J. Spijkman, S.G.J. Mathijssen, R.A.J. Janssen, D.M. de Leeuw, M. Kemerink, Fundamental Limitations for Electroluminescence in Organic Dual-Gate Field-Effect Transistors, Adv. Mater., 26 (2014) 44504455. 


\section{Highlights}

- A general mobility-extraction method is presented

- Mobility in OFETs is determined independent of contact resistances

- Channel currents are induced and probed capacitively with finger-shaped gates

- The method is experimentally and numerically demonstrated

\section{Graphical abstract}
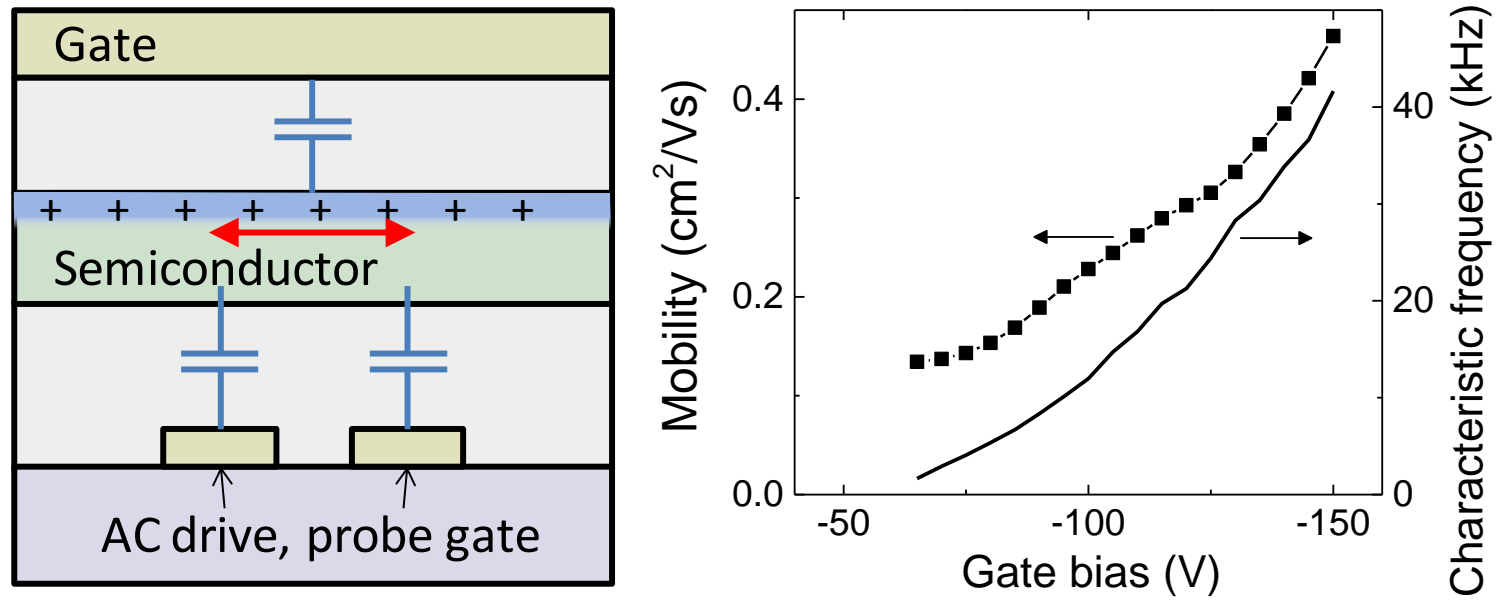